\begin{tabular}{|c|l|}
\hline Title & Strong effects of network architecture in the entrainment of coupled oscillator systems \\
\hline Author(s) & Kori, Hiroshi; Mikhailov, A lexander S. \\
\hline Citation & $\begin{array}{l}\text { Physical Review E, 74, 066115 } \\
\text { https://doi.org/10.1103/PhysRevE.74.066115 }\end{array}$ \\
\hline Issue Date & 2006-12 \\
\hline Doc URL & http://hdl.handle.net/2115/17194 \\
\hline Rights & Copyright $\odot 2006$ A merican Physical Society \\
\hline Type & article \\
\hline File Information & PRE74-6.pdf \\
\hline
\end{tabular}

Instructions for use 


\title{
Strong effects of network architecture in the entrainment of coupled oscillator systems
}

\author{
Hiroshi Kori ${ }^{1, *}$ and Alexander S. Mikhailov ${ }^{2}$ \\ ${ }^{1}$ Department of Mathematics, Hokkaido University, Kita 10, Nishi 8, Kita-Ku, Sapporo, Hokkaido, 060-0810, Japan \\ ${ }^{2}$ Abteilung Physikalische Chemie, Fritz-Haber-Institut der Max-Planck-Gesellschaft, Faradayweg 4-6, 14195 Berlin, Germany
}

(Received 30 August 2006; published 26 December 2006)

\begin{abstract}
Random networks of coupled phase oscillators, representing an approximation for systems of coupled limit-cycle oscillators, are considered. Entrainment of such networks by periodic external forcing applied to a subset of their elements is numerically and analytically investigated. For a large class of interaction functions, we find that the entrainment window with a tongue shape becomes exponentially narrow for networks with higher hierarchical organization. However, the entrainment is significantly facilitated if the networks are directionally biased-i.e., closer to the feedforward networks. Furthermore, we show that the networks with high entrainment ability can be constructed by evolutionary optimization processes. The neural network structure of the master clock of the circadian rhythm in mammals is discussed from the viewpoint of our results.
\end{abstract}

DOI: 10.1103/PhysRevE.74.066115

PACS number(s): 89.75.Fb, 05.45.Xt

\section{INTRODUCTION}

The study of complex networks has applications in various fields including biology and engineering and has attracted growing attention $[1,2]$. In the last decade, much progress has been reached in understanding complexity of network architectures. However, not only the architecture but also the dynamics taking place in a network is important [3]. This dynamics determines functions of the networks, related, e.g., to information processing in the brain (which is a network of neurons) or to the production process in a factory (which is a network of machines). Most of the real-world networks are constructed in such a way that they implement certain desired dynamical behaviors. Not only the individual components of a network (such as single neurons), but also the network architecture (e.g., connections between neurons) should be appropriately designed. Thus, it should be expected that real-world networks have architectures reflecting their desired dynamical behaviors. If one can extract topological properties of the networks relevant for their desired dynamics, this may provide insights into the role of each topological property from the viewpoint of dynamics and help to understand the design principles of functional networks.

In the present study, the focus is on the synchronization of oscillators coupled to complex networks. Synchronization plays a crucial role in the functioning of various systems [4-8]. One of the most intriguing examples is the circadian (i.e., approximately daily) clock in mammals $[9,10]$. The circadian rhythms of the whole body are orchestrated by a central clock in the brain, called the suprachiasmatic nucleus (SCN). This brain tissue is formed by a population of special neurons, known as clock cells. A single clock cell exhibits a robust circadian rhythm in its firing rate, and thus each such cell is a self-sustained oscillator (with oscillations determined by a regulatory loop in a group of genes at the single-cell level). In the SCN, neurons mutually synchronize their physiological rhythms even in the absence of any

\footnotetext{
*Electronic address: kori@nsc.es.hokudai.ac.jp
}

environmental assistance [11], so that this cell population can generate collective periodic signals. Additionally, a different kind of synchronization takes place in the SCN-i.e., entrainment to environmental rhythms. (Here and below, we use the term "entrainment" to describe synchronization to an external input, distinguishing it from autonomous mutual synchronization.) The entrainment of the SCN is essential for the proper functioning of this biological clock. Generally, the intrinsic period of the circadian rhythm is significantly different from $24 \mathrm{~h}$ (in humans, it would typically be $25 \mathrm{~h}$ ). Therefore, it must be tuned to the 24-h period through some external influences. Moreover, the phase of circadian oscillations needs to be locked appropriately to the local time. Such an entrainment process is mediated by the light information coming from the eyes, which acts as external periodic forcing for the SCN. However, only a distinct subset of neurons receives and processes this light information, so that the rest of the population should be indirectly entrained via intercellular communication (see Ref. [9] and references therein). There are various types of intercellular communication inside the SCN, including communication through several types of neurotransmitters [10]. Communication through neurotransmitters goes via chemical synapses of neurons forming a complex directed network.

With respect to mutual synchronization of oscillators, the impact of the network architecture has been intensively investigated in recent years [12-18]. However, only a few studies have dealt so far with the entrainment (i.e., synchronization to external periodic forcing) of oscillator networks [19-21]. The objective of the present paper is to identify principal topological properties that determine the entrainment ability of oscillator networks and to find the dependence of the entrainment ability on these topological properties. Although the problem is motivated by the SCN, our objective is more general. Therefore, we use the phase oscillator model, which provides an approximation for a set of coupled limit-cycle oscillators $[6,22]$. Because of its analytical tractability, we can expect that the phase oscillator model yields general insight into the entrainment phenomena. In an earlier Letter, a particular phase oscillator model has been studied and it was found that, in this model, the entrainment threshold for the coupling intensity between oscillators 
increases exponentially with the depth of a network, defined as the mean forward distance from a pacemaker (i.e., source of external forcing) to the network nodes [20]. Hence, it was found that the entrainment is practically only possible for the shallow networks with low hierarchical organization. Here, we give all details of the derivations, extend the analysis to different network models, and demonstrate the universality of the earlier preliminary results. An additional class of random networks is considered, which allows one further to explore the design principles of entrainable networks. The optimization of the network architecture with respect to the entrainment through a dynamical learning process is further performed.

The article is organized as follows: The model is introduced in Sec. II. Then, we first show numerical results obtained for standard random networks in Sec. III. In Sec. IV, the model is analytically investigated under a certain approximation for the network architecture. The comparison with numerical results is also provided. In Sec. V, a different class of random networks is introduced-i.e., directionally biased networks - and it is shown that the entrainment is strongly enhanced for the networks close to the feedforward type. In Sec. VI, dynamical evolution of the network architecture using two kinds of learning algorithms is considered. The results are discussed, with a special emphasis on the design principles of biological clocks, in Sec. VII, followed by main conclusions in Sec. VIII.

\section{THE MODEL}

We consider a system of $N+1$ phase oscillators, including a special element representing a pacemaker. The basic model is given by a set of evolution equations for the oscillator phases $\phi_{i}(1 \leq i \leq N)$ and the pacemaker phase $\phi_{0}$,

$$
\begin{gathered}
\dot{\phi}_{i}=\omega+\frac{\kappa}{p N} \sum_{j=1}^{N} A_{i j} \Gamma\left(\phi_{i}-\phi_{j}\right)+\mu B_{i} \widetilde{\Gamma}\left(\phi_{i}-\phi_{0}\right), \\
\dot{\phi}_{0}=\omega+\Delta \omega .
\end{gathered}
$$

The network connectivity is determined by the adjacency matrix $\mathbf{A}$, where its elements $A_{i j}$ are either 1 or 0 . Except in Appendix B, we always consider directed networks, so that the matrix $\mathbf{A}$ is asymmetric in general. The mean degree $p N$ is the average number of incoming connections per node (and $p$ is called the connectivity). The pacemaker (i.e., the element with $i=0$ ) has a different frequency $\omega+\Delta \omega$ from those of the all other oscillators, $\omega$. The pacemaker is acting on the oscillators $1 \leq i \leq N_{1}$, the action being specified by the coefficients $B_{i}$ taking 1 for $1 \leq i \leq N_{1}$ and 0 otherwise. The coupling between elements inside the network is characterized by the $2 \pi$-periodic coupling function $\Gamma(x)$ and the (positive) coupling intensity coefficient $\kappa$. In the absence of a pacemaker, such networks undergo autonomous phase synchronization at the natural frequency $\omega$ if the coupling is attracting-i.e., if $\Gamma^{\prime}(0)<0$ (where the prime denotes the derivative). The coupling to the pacemaker is characterized by the $2 \pi$-periodic coupling function $\widetilde{\Gamma}(x)$ and the (positive) intensity coefficient $\mu$. We assume that the functions $\Gamma(x)$ and $\widetilde{\Gamma}(x)$ are nonconstant and smooth.

Without loss of generality, our model can be simplified. By going into a rotating frame, we have $\omega=0$. The maxima of the coupling functions, $\max \Gamma$ and $\max \widetilde{\Gamma}$, are chosen equal to unity by properly defining the coefficients $\kappa$ and $\mu$. Moreover, rescaled time $t^{\prime}=t \Delta \omega$ and rescaled coupling strengths $\kappa^{\prime}=\kappa / \Delta \omega$ and $\mu^{\prime}=\mu / \Delta \omega$ are introduced. ${ }^{1}$ After that, the model takes the form of Eq. (1) with $\Delta \omega=1$ and $\omega=0$ (below, we drop primes in the notation for the rescaled quantities). Note that, in terms of the original model (1), an increase of the rescaled coupling between the elements corresponds either to an increase of coupling $\kappa$ or to a decrease of the relative pacemaker frequency $\Delta \omega$.

The presence of a pacemaker imposes hierarchical organization in the network architecture, which plays a crucial role in determining the entrainment ability. For any node $i$, its distance $l_{i}$ with respect to the pacemaker is defined by the length of the minimum forward path separating this node from the pacemaker. For example, the elements $1 \leq i \leq N_{1}$ (directly connected to the pacemaker) have distances $l_{i}=1$. Among the rest elements, the elements receiving connections from those with distances 1 have distances $l_{i}=2$, etc. Thus, the whole network is divided into a set of shells, each of which is composed of oscillators with distance $h$ from the pacemaker. The shell population $N_{h}$ is given by the number of the oscillators with distance $h$. The depth $L$ of a network is introduced as

$$
L=\frac{1}{N} \sum_{i=1}^{N} l_{i}=\frac{1}{N} \sum_{h} h N_{h}
$$

which is the average distance from the pacemaker to the entire network. This quantity characterizes how hierarchical a given network is. Moreover, we classify the types of connections into forward, backward, and intrashell connections, which are, respectively, connections from the nodes in a certain shell $h$ to the nodes in the next shell $h+1$, from the nodes in a certain shell $h$ to the nodes in the shallower shells $k<h$, and between the nodes inside the same shell. We call the connections coming from a certain node the outgoing connections of the node and the connections received by a certain node the incoming connections of the node.

In the analysis of the model, several further assumptions will be made. The number $N_{1}$ of elements, directly connected to the pacemaker, is assumed to be small as compared with the total size $N$ of the network. The mean degree $p N$ is chosen so large that the networks do not become disconnected

\footnotetext{
${ }^{1}$ We assume here $\Delta \omega>0$. However, the results in the present paper hold also for $\Delta \omega<0$ because of the following reason. The system (1) is invariant under the transformation $\omega \rightarrow-\omega, \Delta \omega \rightarrow-\Delta \omega$, $\phi \rightarrow-\phi, \Gamma(\phi) \rightarrow-\Gamma(-\phi)$, and $\widetilde{\Gamma}(\phi) \rightarrow-\widetilde{\Gamma}(-\phi)$. It will be turned out that the entrainment behavior does not depend on the explicit form of $\Gamma(\phi)$ provided that $\Gamma^{\prime}(0)<0$. Therefore, the same entrainment behavior takes place also for $\Delta \omega<0$. However, in this case, the role of $\max \Gamma$ is replaced by $-\min \Gamma$, so that $-\min \Gamma$ should be put unity by properly rescaling $\kappa$.
} 
and much smaller than the network size $N$. We assume $\Gamma(0)=0$. This assumption implies that coupling between connected oscillators vanishes when their phases are synchronized, as is often the case in various systems (e.g., diffusive or gap-junction couplings). Moreover, we assume the limit $\mu \rightarrow \infty$ in the present paper except in Sec. IV B. Under such an assumption, instead of Eq. (1) we use the following model:

$$
\begin{gathered}
\phi_{i}=t \quad \text { for } 1<i<N_{1}, \\
\dot{\phi}_{i}=\frac{\kappa}{p N} \sum_{j=1}^{N} A_{i j} \Gamma\left(\phi_{i}-\phi_{j}\right) \quad \text { for } N_{1}<i<N .
\end{gathered}
$$

Only in Sec. IV B do we investigate the original model (1) to obtain the entrainment condition for $\mu$.

\section{NUMERICAL INVESTIGATIONS FOR STANDARD RANDOM NETWORKS}

We begin our analysis of entrainment phenomena by considering the important special case of standard random networks, also known as Erdös-Rényi (ER) networks [23,24]. These networks are generated by independently assigning with probability $p$ for any pair $i$ and $j$ of the network nodes a connection that leads from the node $i$ to the node $j$. Hence, elements $A_{i j}$ of the adjacency matrix $\mathbf{A}$ are chosen to be 1 with probability $p$ and 0 otherwise. Note that $\mathbf{A}$ is asymmetric in general (representing a directed network). Only sparse random networks with a small mean degree $p N \ll N$ will be considered.

We numerically investigate the model (3) (corresponding to the limit $\mu \rightarrow \infty$ ) with the following coupling function:

$$
\Gamma(x)=-\frac{\sin (x+\alpha)-\sin \alpha}{1+\sin \alpha},
$$

where $\alpha$ is constant. Note that for any $\alpha \Gamma(0)=0$ is 0 and $\Gamma(x)$ is normalized such that $\max \Gamma(x)=1$. Note also that for $\alpha=0$ this coupling function is the same as that used in Ref. [20].

Numerical simulations are started with random phases for the oscillators $i>N_{1}$. For each oscillator, its effective long-time frequency $\omega_{i}$ is computed as

$$
\omega_{i}=\frac{\phi_{i}\left(t_{0}+T\right)-\phi_{i}\left(t_{0}\right)}{T},
$$

with sufficiently large $T$ and $t_{0}$.

Numerical simulations show that the response of a network to the introduction of a pacemaker depends on the strength $\kappa$ of coupling between the oscillators. When this coupling is sufficiently large, the pacemaker entrains the whole network (i.e., $\omega_{i}=1$ for any $i$ ). Under entrainment, the relative phases $\phi_{i}-\phi_{0}$ are locked. As the coupling strength $\kappa$ is decreased, the entrainment breaks down at a certain threshold value $\kappa_{\text {cr }}$ (for $\alpha=0$; see Fig. 1). Our simulations show that, in most cases, synchronization between the first and second shells is the first to break down and the frequencies of oscillators in the shells $h \geq 2$ remain equal for any $\kappa$-i.e.,

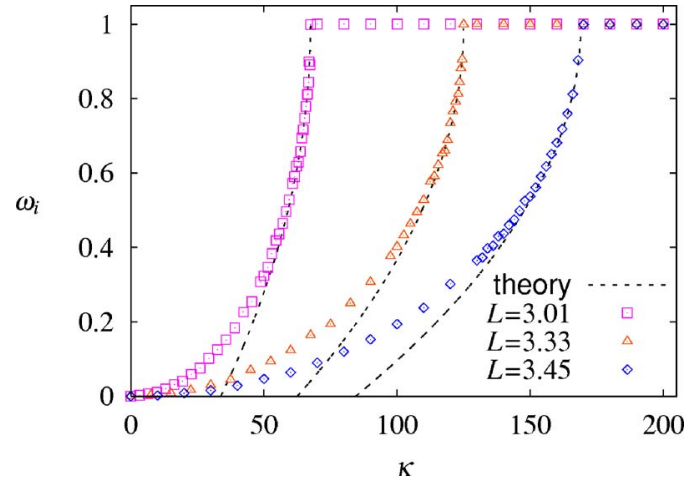

FIG. 1. (Color online) Long-time frequencies for various $\kappa$. Data obtained for three samples of random networks are plotted with different symbols. The depths of the networks are $L=3.01$, 3.33, and 3.45. The lines show the theoretical curves (32) with $\kappa_{\mathrm{cr}}=67.5,125$, and 169 , respectively. $N=100, p=0.1, N_{1}=1$, and $\alpha=0$.

$\omega_{i}=\omega_{j}$ for $i, j>N_{1}$. The results for $\alpha=0.5$ and $\alpha=-0.5$ are similar.

Figure 2 displays the thresholds $\kappa_{\mathrm{cr}}$ (in the logarithmic scale) for a large set of networks with the fixed mean degree $p N$ and different numbers $N_{1}$ of oscillators in the first shell. As can be seen, the entrainment threshold $\kappa_{\text {cr }}$ may vary largely for different network realizations even if $N_{1}$ is fixed. The results for $\alpha=-0.5,0$, and 0.5 are labeled as (A), (B), and $(\mathrm{C})$. The same set of networks is used for all three values of $\alpha$. For a given network, the entrainment thresholds obtained for different values of $\alpha$ are close, implying that the entrainment threshold does not depend on the particular form of the coupling function. Each group of networks with a certain $N_{1}$ is displayed with its own symbol. Each group is characterized by a distribution of depths $L$ and generates therefore a cluster of data points. Correlation between the entrainment threshold $\kappa_{\mathrm{cr}}$ and the network depth $L$ is evident even within a fixed $N_{1}$. The distributions inside each cluster and the accumulation of the clusters yield the dependence $\kappa_{\mathrm{cr}}(L)$ of the entrainment threshold on the network depth. Remarkably, the observed dependence is well numerically approximated by the exponential law

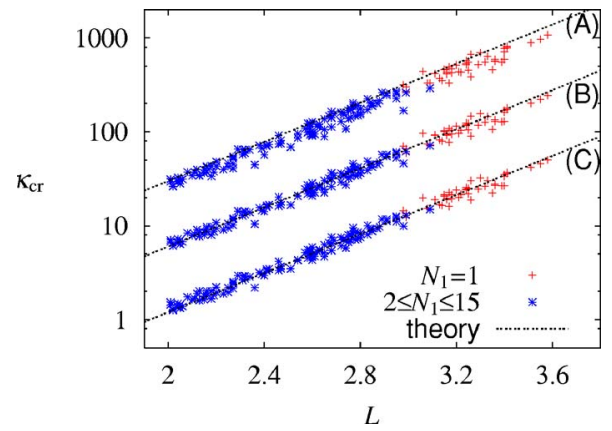

FIG. 2. (Color online) Dependences of the entrainment threshold on the depth $L$ for a large set of random networks of size $N=100$ and $p N=10$. The coupling function is given by Eq. (4) with (A) $\alpha=0.5$, (B) $\alpha=0$, and (C) $\alpha=-0.5$. To separate the data sets, here we have plotted $\nu \kappa_{\mathrm{cr}}$ with $\nu=5,1$, and 0.2 , respectively, for (A), (B), and (C). The lines are the theoretical dependence $c \nu \kappa_{\mathrm{cr}}$, where $\kappa_{\mathrm{cr}}$ is given by Eq. (33) and $c=0.60$ is a fitting parameter. 

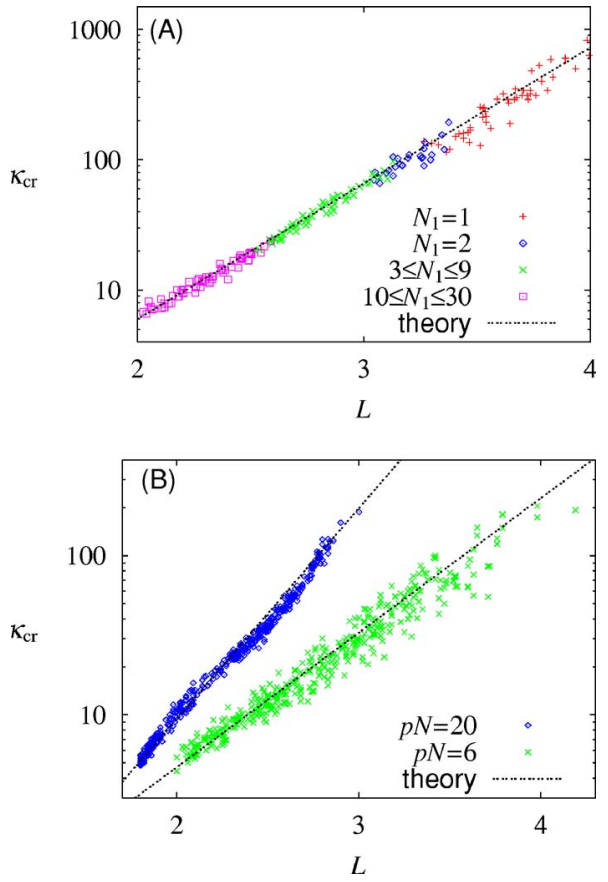

FIG. 3. (Color online) Dependences of the entrainment threshold on the depth $L$ for a large set of random networks. The coupling function is given by Eq. (4) where $\alpha=0$. (A) $N=200$ and $p N=10$. The line is the theoretical dependence $c \kappa_{\mathrm{cr}}$, where $\kappa_{\mathrm{cr}}$ is Eq. (33) and $c=0.60$ (the same as the value used for $N=100$ ). (B) $N=100$ and $p N=6,20$. The lines are the theoretical dependence $c \kappa_{\mathrm{cr}}$, where $\kappa_{\text {cr }}$ is Eq. (33) and $c=0.67$ and 0.45 , respectively, for $p N=6$ and 20.

$$
\kappa_{\mathrm{cr}} \propto(1+p N)^{L} .
$$

The same functional dependence is found for networks of other system sizes and different average mean degrees $p N$ (see Fig. 3). The entrainment thresholds for $N=100$ and $N=200$ can be well fitted by the same function with the same fitting parameter $c$, which suggests that the entrainment threshold depends not explicitly on the system size $N$, but on the average degree $p N$.

Next we consider relaxation to the entrained state. The relaxation time for each generated network has been measured as follows. First, we have run a numerical simulation for a long time starting with a random initial distribution of phases. If the relative phases $\psi_{i} \equiv \phi_{i}-\phi_{0}$ for all $i$ were not locked at the end of the simulation (implying that the network did not become entrained for the chosen coupling intensity), such a network has been discarded. Then, the simulation was repeated starting from the initial condition $\phi_{i}(t=0)=\psi_{i}^{\text {st }}+\epsilon y_{i}$ for $i>N_{1}$, where $y_{i}$ is a random number independently taken from the uniform distribution within $(0,1), \epsilon$ is a small coefficient, and $\psi_{i}^{\text {st }}$ are the relative phases of oscillators in the entrained state. The time dependence of the distance $D(t) \equiv \Sigma_{i} \sqrt{\left\{\psi_{i}(t)-\psi_{i}^{\text {st }}\right\}^{2}}$ has been monitored. The relaxation time $\tau$ was defined as the time $t$ at which the ratio $D(t) / D(0)$ becomes equal to $e^{-1}$. Figure 4 displays the relaxation times $\tau$ (in the logarithmic scale), obtained numerically for a large set of networks and for a fixed coupling strength $\kappa$. Again, the exponential dependence on the depth is evident. This dependence is well fitted by the function

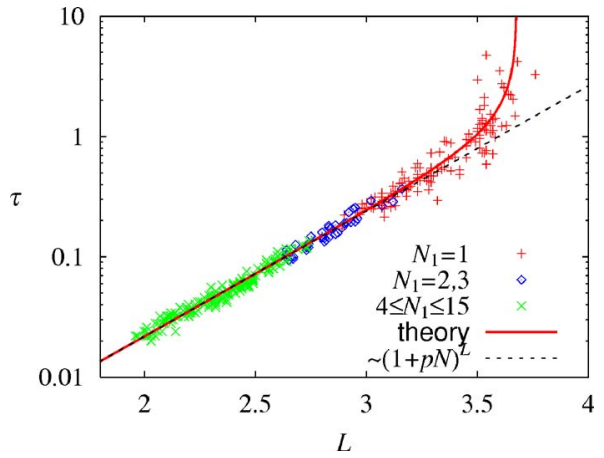

FIG. 4. (Color online) Dependence of the relaxation time on the depth $L$ for an ensemble of random networks. The parameters are $N=100, p=0.1, \alpha=0$, and $\kappa=300$. The solid line is the theoretical dependence $d \tau$ where $\tau$ is Eq. (28) with $\kappa_{\mathrm{cr}}=0.6 p N(1+p N)^{L-2}$ (same as the theoretical lines in Fig. 2) and $d=1.22$ is an additional fitting parameter. The dotted line is the exponential function $\kappa_{\mathrm{cr}} / \kappa \propto(1+p N)^{L}$.

$$
\tau_{\mathrm{cr}} \propto(1+p N)^{L} .
$$

There is a divergence of the relaxation time around $L=3.7$. This divergence occurs around the region of the depth where given coupling strength $\kappa$ is close to the entrainment threshold-i.e., $\kappa \simeq \kappa_{\mathrm{cr}}(L)$. Above this region, entrainment rarely happens.

The eigenvalues of an entrained state are displayed in Fig. 5 . They were obtained by numerically solving the stability matrix of our model (3). We have assumed the limit $\mu \rightarrow \infty$ and perturbations are considered only in the subsystem $i>N_{1}$, so that there are $N-N_{1}$ eigenvalues. The eigenvalue possessing the maximum real part is denoted by $\lambda_{\max }$. As seen, the magnitude of the real part of this eigenvalue, $\left|\operatorname{Re} \lambda_{\max }\right|$, is much smaller than those of others (which are distributed around $|\kappa|$ ). We have preliminarily checked the eigenvectors and found that the associated eigenvector with $\lambda_{\max }$ corresponds to an approximately identical phase shift of the whole subsystem $i>N_{1}$ and the remaining eigenvectors correspond to relative motions inside the subsystem. This fact suggests the following relaxation process: Relative

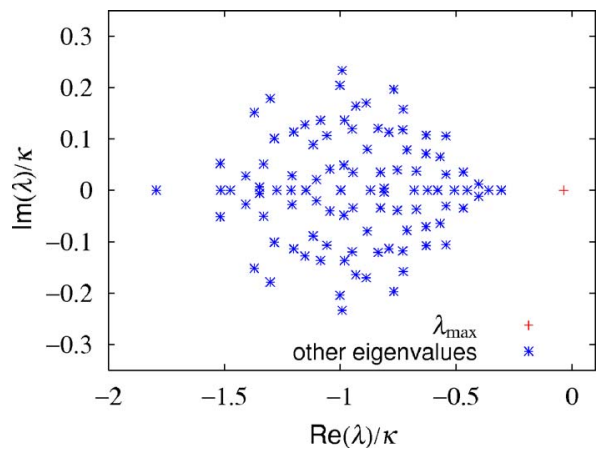

FIG. 5. (Color online) Eigenvalues (divided by $\kappa$ ) of the entrained state. The parameters are $N=100, N_{1}=2, p=0.1, \alpha=0$, and $\kappa=300$ (the depth of the used network is $L=2.86$ and the entrainment threshold for this network is found numerically to be $\kappa_{\mathrm{cr}}$ $\simeq 33$ ). The real part of the maximum eigenvalue is $\operatorname{Re}\left(\lambda_{\max }\right)-11$. 


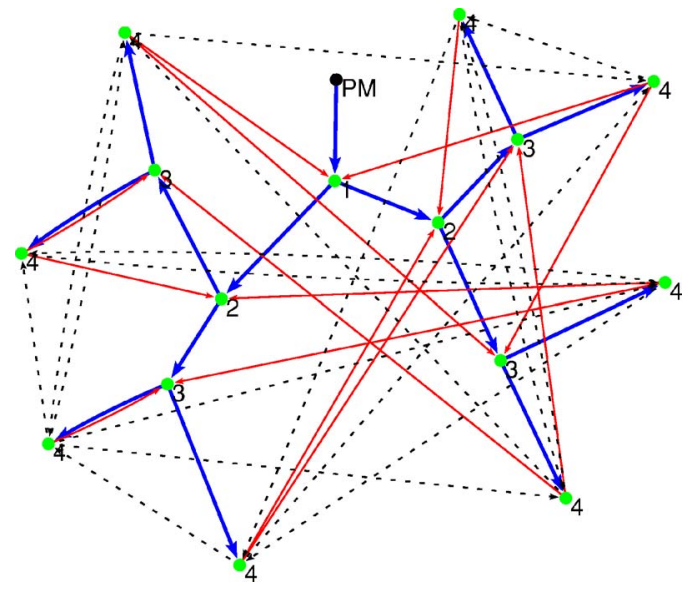

FIG. 6. (Color online). An example of a global tree network $\left(N_{1}=1, p N=2, H=4\right.$, and hence, $\left.N=15\right)$. Thick solid lines, thin solid lines, and dotted lines represent, respectively, forward connections, backward connections, and intrashell connections. Numbers indicate the hierarchical level of each node. This graph was constructed using the PAJEK software package.

perturbations inside the subsystem quickly diminish within the characteristic time scale $\kappa^{-1}$ and, then, the phase differences between the oscillators in the subsystem become practically locked. The relaxation of the phase difference between the first shell and the subsystem proceeds slowly with the time scale $\left|\operatorname{Re} \lambda_{\max }\right|^{-1}$. Such behavior has actually been observed in our numerical simulations.

The results relating to the relaxation process do not change qualitatively for different values of $\alpha$ (we have further checked them for $\alpha=0.5$ and $\alpha=-0.5)$. Note also that the relaxation time does not depend on the particular form of small perturbations.

\section{ANALYTICAL INVESTIGATIONS IN THE GLOBAL TREE APPROXIMATION}

In this section, the model is analytically investigated using the heuristic global tree approximation. First, global tree networks will be introduced and then their similarity to the ER networks will be explained. After that, the analytical solution of the entrainment problem for global tree networks will be constructed. Note that this analytical solution will hold only in the limit when both $p N$ and $N$ are large.

\section{A. Global tree approximation}

A global tree network is a hierarchical network where, in any level, each oscillator has only one incoming connection from the higher level and exactly $p N$ outgoing connections leading to the lower level. Additionally, each oscillator has exactly $p N$ connections from the last (bottom) shell of this hierarchical network. No other connections exist, and the precise connection topology remains arbitrary. An example of a global tree network with four hierarchical levels is shown in Fig. 6.

For such networks, shell populations grow exponentially-i.e., as $N_{h}=N_{1}(p N)^{h-1}$ — with distance $h$ from the network origin. The total number $H$ of shells is determined by the condition $\Sigma_{h=1}^{H} N_{h}=N$. The depth of such a network is given by $L=N^{-1} \sum_{h=1}^{H} h N_{h}=H+O(1 / p N)$. Thus, for $p N \rightarrow \infty$, the depth $L$ coincides with the number of shells, $H$. Therefore, $H$ can be approximately replaced by $L$.

Global tree networks share essential properties with the ER networks of large size $N \gg 1$ and high connectivity $p N \gg 1$. This similarity is briefly explained below (see Appendix A for a detailed discussion). We first consider the pattern of forward connections of the ER network. By definition of our model, each node in the first shell receives one forward connection from the pacemaker. Each node typically gives $p N$ outward connections. Thus, from the first shell, a total number of the outward connections is $p N N_{1}$, on average. All elements receiving a connection from the first shell form the second shell of the network. If the number of connections leading from the first shell is much smaller than the total number $N$ of elements to which they may lead (i.e., if $\left.p N N_{1} \ll N\right)$, each next outward connection from the first shell is typically received by a different node in the second shell. This means that, typically, an element in the second shell would be linked only to a single element in the first shell, as required by the tree structure. Considering the third shell, we can notice again that, if the number $N_{3}=N_{1}(p N)^{2}$ of outgoing connections from this shell is small as compared with the network size $N$, the tree structure would approximately hold for this shell too.

Shell populations $N_{h}$ grow exponentially with the number $h$ of the shell-i.e., $N_{h}=N_{1}(p N)^{h-1}$. The tree structure with respect to forward connections holds as far as these populations remain much smaller than the total network size. When $p N$ is large, it can be shown that only the last two shells have populations of size $O(N)$ and, thus, the tree structure approximately holds down to the third last shell. Analyzing further patterns of backward and intrashell connections in a large ER network, we notice that the populations of all shells, except the last of them, are of order $o(N)$ and only the populations of the two last shells are of order $O(N)$. Therefore, each node outside of the last two shells receives backward connections mostly from these last two shells. On average, the number of such connections is $p N$. Each node in the last two shells typically has $p N$ incoming connections from the last two shells. This large number of connections between the last two shells strongly facilitates synchronization of oscillators inside them, as can be seen in numerical simulations. Having this in mind, we may merge the last two shells in the network into a single shell. Once this additional (empirical) approximation is used, the tree structure is extended to the entire network, including its last shells.

Thus, in the global tree approximation the ER network is treated as a network having a tree structure with respect to forward connections and with the backward connections arriving only from the last network shell. In this network, every forward path starting from the pacemaker has a similar structure, as illustrated in Fig. 7.

When this approximation is used, an analytical solution can be easily constructed and its properties become more clear. Therefore, we prefer first to present our analysis using the global approximation, even if it remains partly empirical. 


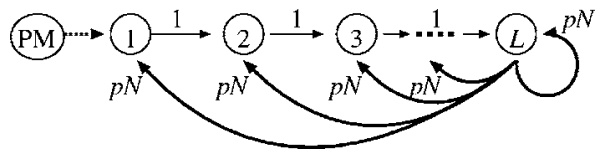

FIG. 7. Schematic representation of the network structure along a forward path in the global tree approximation. Each circle with a number $h$ is an oscillator of the shell $h$. Each right arrow and each left arrow represent, respectively, a forward connection and a $p N$ backward (or intrashell) connection.

The investigation of ER networks can also be performed without relying on the tree approximation. While giving quantitatively better results, this other analytical investigation is, however, more complicated and, therefore, we give it separately in Appendix A.

\section{B. Entrained solution}

In the considered tree networks, our model (1) can be written as

$$
\begin{gathered}
\dot{\phi}_{i}^{1}=\mu \widetilde{\Gamma}\left(\phi_{i}^{1}-t\right)+\frac{\kappa}{p N} \sum_{j} A_{i j} \Gamma\left(\phi_{i}^{1}-\phi_{j}^{L}\right), \\
\dot{\phi}_{i}^{h}=\frac{\kappa}{p N} \Gamma\left(\phi_{i}^{h}-\phi_{k}^{h-1}\right)+\frac{\kappa}{p N} \sum_{j} A_{i j} \Gamma\left(\phi_{i}^{h}-\phi_{j}^{L}\right),
\end{gathered}
$$

where $h \geq 2, \phi_{i}^{h}$ is the phase of the oscillator $i$ in the shell $h$, $\phi_{k}^{h-1}$ is the oscillator giving forward connection to the oscillator $i$ in the shell $h$, and the summation is taken over all the oscillator $j$ in the last shell $L$. Because of the assumed global tree structure, each oscillator in any particular shell has the same pattern of connections-i.e., one forward connection from the previous shell and $p N$ backward connections from the last shell. Thus, the entrained state of a network with phase synchronization inside every shell is possible. For such a state, the entrainment solution problem is reduced into that in the one-dimensional oscillator array along a forward path starting from the pacemaker (see Fig. 7). Putting $\dot{\phi}_{i}=1$ (which is the entrainment condition) and $\phi_{i}^{h}=\theta_{h}$ for all the oscillator $i$ in the shell $h$, we get the following algebraic equations:

$$
\begin{gathered}
\mu \widetilde{\Gamma}\left(\theta_{1}-t\right)+\kappa \Gamma\left(\theta_{1}-\theta_{L}\right)=1, \\
\frac{\kappa}{p N} \Gamma\left(\theta_{h}-\theta_{h-1}\right)+\kappa \Gamma\left(\theta_{h}-\theta_{L}\right)=1 \quad \text { for } h \geq 2 .
\end{gathered}
$$

For large $p N$, we may linearize $\Gamma\left(\theta_{h}-\theta_{k}\right)$ as $\Gamma^{\prime}(0)\left(\theta_{h}-\theta_{k}\right)$ for $h, k \geq 2$ [it will be shown that $\theta_{2}-\theta_{L}$ is at most of order $O(1 / p N)$ in the solution under entrainment, so that this linearization is justified]. We may then solve Eq. (10) from the last shell upward. For $h=L$, we get

$$
\Gamma\left(\theta_{L}-\theta_{L-1}\right)=\kappa^{-1} p N .
$$

Substitution of this into Eq. (10) for $h=L-1$ results in

$$
\Gamma\left(\theta_{L-1}-\theta_{L-2}\right)=\kappa^{-1} p N(1+p N) .
$$

From now on, the linear approximation is employed. We may then get the following equation successively from $h=L-2$ to $h=2$ :

$$
\Gamma\left(\theta_{h}-\theta_{h-1}\right)=\kappa^{-1} p N(1+p N)^{L-h} \text { for } h \geq 2 .
$$

Substituting Eq. (13) for $h=2$ into Eq. (9), we also obtain

$$
\widetilde{\Gamma}\left(\theta_{1}-t\right)=\frac{1-\kappa \Gamma\left(\theta_{1}-\theta_{L}\right)}{\mu} \leq \frac{1-\kappa \min \{\Gamma(x)\}}{\mu} .
$$

Note that an explicit expression for $\widetilde{\Gamma}\left(\theta_{1}-t\right)$ is not needed.

The existence conditions of the entrained solution are $\widetilde{\Gamma}\left(\theta_{1}-t\right) \leq 1$ and $\Gamma\left(\theta_{h}-\theta_{h-1}\right) \leq 1$ for $h>2$. The former condition is satisfied for $\mu \gg \kappa$ (which is our assumption). Among the terms $\Gamma\left(\theta_{h}-\theta_{h-1}\right)$, the term $\Gamma\left(\theta_{2}-\theta_{1}\right)$ is the largest one. The solution thus exists if

$$
\Gamma\left(\theta_{2}-\theta_{1}\right) \leq 1 \text {. }
$$

Therefore, the entrained state of the network is possible only if the coupling intensity $\kappa$ satisfies the condition $\kappa \geq \kappa_{\mathrm{cr}}$, where

$$
\kappa_{\mathrm{cr}}=p N(1+p N)^{L-2} .
$$

The result (16) is remarkable. According to it, the coupling threshold $\kappa_{\mathrm{cr}}$ is determined exclusively by topological properties and does not depend on a particular form of the coupling function. Moreover, it is given only through a combination of $p N$ and $L$. This fact suggests that the essential parameters of the system are $p N$ and $L$ rather than $N, p$, and $N_{1}$, in agreement with the previous numerical results obtained for the ER networks.

From Eq. (16), $\kappa_{\mathrm{cr}}$ for our tree network under consideration is roughly estimated as

$$
\kappa_{\mathrm{cr}} \sim(p N)^{L-1} \sim N / N_{1} .
$$

Thus, $\kappa_{\mathrm{cr}}$ is a large number for small $N_{1}$ (as we assumed). This property will be used in the derivation of the relaxation time.

One can verify that $\theta_{2}-\theta_{L}$ is at most of order $(p N)^{-1}$ in the entrained state by substituting $\kappa_{\mathrm{cr}}$ into $\kappa$ in Eq. (13). Note also that although the phase differences $\theta_{h}-\theta_{h+1}$ are small in deeper shells, they should not be neglected when the entrainment threshold is derived, because the phase difference $\theta_{1}-\theta_{2}$ (which determines the existence condition of the entrained solution) is a consequence of the exponential growth of such small phase differences from the deepest shell upwards.

\section{Stability analysis of the entrained state}

To analyze stability of the entrained solution, only the limit $\mu \rightarrow \infty$ is considered, and thus, the model (3) is used. In numerical simulations, we have observed that the relaxation process is characterized by two distinct time scales: the first one characterizes fast relaxation inside the subsystem $i>N_{1}$ and the other corresponds to slow relaxation in the phase difference between the first shell and the subsystem. In this 
section, we first investigate the internal stability of the subsystem. Then, taking advantage of the separation of time scales, we heuristically construct the effective dynamical equation of the whole subsystem.

We consider small perturbations for phases in the entrained state-i.e., $\phi_{i}^{h}=\theta_{h}+\delta \phi_{i}^{h}$ where $\delta \phi_{i}^{h}$ is a small perturbation. Because of the assumption $\mu \rightarrow \infty$, we put $\delta \phi_{i}^{1}=0$-i.e., $\phi_{i}^{1}=\theta_{1}=t$. The model (8) for $h \geq 2$ then gives

$$
\begin{aligned}
\delta \dot{\phi}_{i}^{h}= & \frac{\kappa}{p N} \Gamma^{\prime}\left(\theta_{h}-\theta_{h-1}\right)\left(\delta \phi_{i}^{h}-\delta \phi_{k}^{h-1}\right) \\
& +\frac{\kappa}{p N} \sum_{j} A_{i j} \Gamma^{\prime}\left(\theta_{h}-\theta_{L}\right)\left(\delta \phi_{i}^{h}-\delta \phi_{j}^{L}\right) .
\end{aligned}
$$

Because $\theta_{h}-\theta_{L}=O(1 / p N)$, we approximate $\Gamma^{\prime}\left(\theta_{h}-\theta_{L}\right)$ $\simeq \Gamma^{\prime}(0)$. In addition, because of the large number $p N$ of connections from the last shell, we may approximate

$$
\frac{1}{p N} \sum_{j} A_{i j} \delta \phi_{j}^{L} \simeq \frac{1}{N_{L}} \sum_{j} \delta \phi_{j}^{L} \equiv \overline{\delta \phi^{L}} .
$$

Using these two approximations, Eq. (18) reduces to

$$
\delta \dot{\phi}_{i}^{h}=\frac{\kappa}{p N} \Gamma^{\prime}\left(\theta_{h}-\theta_{h-1}\right)\left(\delta \phi_{i}^{h}-\delta \phi_{k}^{h-1}\right)+\kappa \Gamma^{\prime}(0)\left(\delta \phi_{i}^{h}-\overline{\delta \phi^{L}}\right) .
$$

Since the first term is negligibly small for large $p N$ for any shell $h \geq 2$, Eq. (20) is further approximated as

$$
\delta \dot{\phi}_{i}^{h} \simeq \kappa \Gamma^{\prime}(0)\left(\delta \phi_{i}^{h}-\overline{\delta \phi^{L}}\right) .
$$

Thus, in our approximation, all eigenvalues associated with the relative motion inside the subsystem are degenerated into

$$
\lambda=\kappa \Gamma^{\prime}(0),
$$

with degeneracy $N-N_{1}-1$. The dynamical equation (21) describes the fast relaxation inside the subsystem: oscillators quickly relaxes to the average perturbation $\overline{\delta \phi^{L}}$ in the last shell-i.e., $\phi_{i}^{h} \rightarrow \theta_{h}+\overline{\delta \phi^{L}}$. After this fast relaxation, the phase differences inside the subsystem are almost locked.

Now we heuristically construct the dynamical equation for the phase difference between the first shell and the subsystem. We take the coordinate of the subsystem on its surface-i.e., the phase of the second shell-defined as $\Psi=\theta_{2}+\overline{\delta \phi^{L}}$. Because the total external force applied to the subsystem is given by $N_{2} \kappa \Gamma(\Psi-t) / p N=N_{1} \kappa \Gamma(\Psi-t)$, the effective dynamical equation for the surface $\Psi$ reads

$$
N_{\mathrm{e}} \dot{\Psi}=N_{1} \kappa \Gamma(\Psi-t) .
$$

Here, the effective size $N_{\mathrm{e}}$ of the whole network is determined by the condition that $\Psi=\theta_{2}$ when the subsystem is entrained (i.e., $\dot{\Psi}=1)$. From this condition, comparing Eq. (13) for $h=2$ and Eq. (23), we obtain

$$
N_{\mathrm{e}}=N_{1} \kappa_{\text {cr }} .
$$

Thus, from Eqs. (23) and (24) the last eigenvalue is found to be

$$
\lambda_{\max }=\frac{\kappa \Gamma^{\prime}\left(\theta_{2}-\theta_{1}\right)}{\kappa_{\mathrm{cr}}} .
$$

The eigenvector of $\lambda_{\max }$ corresponds to the identical phase shift for all the oscillators $i>N_{1}$.

For the phase difference $\theta_{2}-\theta_{1}$, there are at least one pair of steady solutions, one of which always satisfies $\Gamma^{\prime}\left(\theta_{2}\right.$ $\left.-\theta_{1}\right)<0$. Thus, provided that $\Gamma^{\prime}(0)<0$, a stable entrained solution exists for $\kappa<\kappa_{\mathrm{cr}}$. This implies that the entrainment breakdown occurs only via the disappearance of the solution at $\kappa=\kappa_{\text {cr }}$.

Because $\kappa_{\mathrm{cr}}$ is a large number for small $N_{1}$ [see Eq. (17) ], the time scales are well separated-i.e., $\left|\lambda_{\max }\right| \ll|\lambda|$. This fact justifies the approximation employed in this subsection. In addition, the relaxation time against any general perturbation is thus given simply by

$$
\tau=\left|\lambda_{\max }\right|^{-1}=-\frac{\kappa_{\mathrm{cr}}}{\kappa \Gamma^{\prime}\left(\theta_{2}-\theta_{1}\right)} .
$$

Hence, in general, the relaxation time depends on the explicit form of the coupling function, being different from the case of the entrainment threshold. Nevertheless, the dependence on $L$ is approximately the same as $\kappa_{\mathrm{cr}}$ in the region of $L$ where $\Gamma^{\prime}\left(\theta_{2}-\theta_{1}\right)$ does not vary much with $L$.

For the special case $\Gamma(x)=-\sin x$, Eq. (25) reduces to

$$
\lambda_{\max }=-\frac{\sqrt{\kappa^{2}-\kappa_{\mathrm{cr}}^{2}}}{\kappa_{\mathrm{cr}}} .
$$

The relaxation time is thus

$$
\tau=\frac{\kappa_{\mathrm{cr}}}{\sqrt{\kappa^{2}-\kappa_{\mathrm{cr}}^{2}}} .
$$

Because $\kappa_{\text {cr }}$ increases with $L, \kappa_{c r}$ eventually coincides with $\kappa$ at certain critical $L$, which results in the divergence of $\tau$. In the other region of $L$, the dependence of the relaxation time on the depth $L$ is approximately the same as $\kappa_{\mathrm{cr}}$-i.e., exponential.

\section{Below the entrainment threshold}

The dynamical behavior just below the entrainment threshold is considered. We again choose the limit $\mu \rightarrow \infty$. Because of the property $\left|\lambda_{\max }\right| \ll|\lambda|$, we only need to consider the dynamics of the subsystem, Eq. (23). We introduce the slow mode $x \equiv \Psi-t$ and the bifurcation parameter $\epsilon \equiv\left(\kappa-\kappa_{\mathrm{cr}}\right) / \kappa_{\mathrm{cr}}$. Substituting them into Eq. (23), we obtain

$$
\dot{x}=(1+\epsilon) \Gamma(x)-1 .
$$

Expansion of $\Gamma(x)$ up to the second order around its maximum $x=x_{\max }$ yields

$$
\dot{x}=\epsilon+\frac{\Gamma_{\max }^{\prime \prime}}{2}\left(x-x_{\max }\right)^{2}+\text { higher orders, }
$$

where $\Gamma_{\max }^{\prime \prime}<0$ is the second derivative of $\Gamma(x)$ at $x=x_{\max }$. The steady solution of $x$ (corresponding to the entrainment) thus disappears via a saddle-node bifurcation at $\epsilon=0$, and $x$ oscillates with the negative frequency $\omega_{x}$ for $\epsilon<0$. This 
frequency is found through the integration of Eq. (30):

$$
\frac{2 \pi}{\omega_{x}}=\int_{0}^{2 \pi} \frac{d x}{\epsilon+\Gamma_{\max }^{\prime \prime}\left(x-x_{\max }\right)^{2} / 2}+O(\epsilon),
$$

followed by $\omega_{x}=-\sqrt{2 \epsilon / \Gamma_{\text {max }}^{\prime \prime}}$. Hence, the effective frequency $\omega_{i}$ of each oscillator in the subsystem $\left(i>N_{1}\right)$ for $\epsilon<0$ is

$$
\omega_{i}=1-\sqrt{\frac{2 \epsilon}{\Gamma_{\max }^{\prime \prime}}}+O(\epsilon) .
$$

\section{E. Comparison with numerical results}

The analytical results obtained under the global tree approximation are compared with the numerical results obtained for the ER networks. The theoretical curves plotted in Fig. 1 correspond to the function (32) for appropriate values of $\kappa_{\mathrm{cr}}$, showing excellent agreement with the numerical data for $\kappa$ close to $\kappa_{\mathrm{cr}}$, as expected. In Figs. 2 and 3, we display

$$
\kappa_{\mathrm{cr}}=c p N(1+p N)^{L-2},
$$

where $c$ is a fitting parameter added to our theoretical dependence (16). As already noticed in Ref. [20], although the principal dependence on the depth, $(1+p N)^{L}$, is correctly reproduced by the global tree approximation, the coefficient is somewhat different. We have thus introduced the fitting parameter $c$. As seen in Figs. 2 and 3, the principal dependence on the depth obtained for the tree network agrees excellently with the numerical results obtained for the ER networks. Note that the theoretical dependence, analytically derived directly for the ER network in Appendix A, agrees well with numerical data without any fitting parameter. For the relaxation time, taking into account the correction for $\kappa_{\mathrm{cr}}$, we use instead of Eq. (28) the following function:

$$
\tau=d \frac{c \kappa_{\mathrm{cr}}}{\sqrt{\kappa^{2}-\left(c \kappa_{\mathrm{cr}}\right)^{2}}},
$$

where the value of $c$ is obtained by numerical fitting for the entrainment threshold and $d$ is an additional fitting parameter. As seen in Fig. 4, the function (34) fits very well to numerical data. In Fig. 5, real parts of the eigenvalues except $\lambda_{\text {max }}$ are scattered around $\kappa$, as expected from Eq. (22) [note that $\Gamma^{\prime}(0)=1$ for $\alpha=0$ ]. Putting $\kappa=300$ and $\kappa_{\text {cr }}=33$ into Eq. (27), we obtain $\lambda_{\max } \simeq-9.0$, which is close to the numerical result shown in Fig. $5\left(\lambda_{\max } \simeq-11\right)$.

\section{ENTRAINMENT IN DIRECTIONALLY BIASED NETWORKS}

We can understand by looking at Eq. (10) why entrainment is difficult in the networks with larger depths. The first term on the left side in the equation describes the force of the forward connection. Its sign is positive, so that it contributes to the entrainment. On the other hand, the second term describes the force from the backward connections, whose sign is negative. By moving this second term to the right side, it is seen that it essentially increases the frequency to which the oscillators synchronize. In other words, the backward connections act as a load for the entrainment. Moreover, the number $(p N)$ of backward connections is much larger than the number (1) of forward connections. Thus, to compensate such a strong unbalance, the phase difference associated with the forward connection $\left(\theta_{h-1}-\theta_{h}\right)$ needs to be much larger that associated with the backward connections $\left(\theta_{h}-\theta_{L}\right)$. The effect accumulates exponentially along a forward path of the length $L$ starting from the pacemaker and ending at the last shell. This accumulation results in the exponential growth of the phase difference from the last shell upwards and, thus, the exponential dependence of the entrainment threshold on the depth $L$. Hence, due to the large number of backward connections, the entrainment is very difficult for networks with large depths.

It is thus expected that the entrainment threshold decreases significantly for networks that are closer to the feedforward architecture (i.e., to a network without backward connections). This is indeed demonstrated below using a special class of random networks which we call directionally biased networks. To construct a directionally biased network, we first generate a directed ER random network and choose $N_{1}$ nodes as the first shell. Then, we redefine its backward connections. Namely, for every backward connection, we decide to retain it with probability $\xi$ or delete it otherwise. We call $\xi$ the backward connectivity of the network. Note that a directed ER network corresponds to $\xi=1$ and the feedforward network is obtained for $\xi=0$.

To solve the entrainment problems for such networks, the same approximations as in Sec. IV are applied. The global tree approximation for the forward connection pattern can be used. Furthermore, it can be assumed that all backward connections come from the last shell and that the number of such connections received by a oscillator is $\xi p N$. The former approximation is applicable when $p N$ is large, and the latter is applicable when $\xi p N$ is large. Thus, we require that $\xi p N$ is large. Under this condition, the same linear approximation as in Sec. IV can be used, because the phase difference $\theta_{2}-\theta_{L}$ turns out to be at most of order $(\xi p N)^{-1}$. For simplicity, we further assume $\mu \rightarrow \infty$ also in this section. Then, the entrained solution for the approximated network is found by solving the algebraic equations

$$
\frac{\kappa}{p N} \Gamma\left(\theta_{h}-\theta_{h-1}\right)+\kappa \xi \Gamma\left(\theta_{h}-\theta_{L}\right)=1 \quad \text { for } h \geq 2 .
$$

We obtain

$$
\begin{gathered}
\Gamma\left(\theta_{h}-\theta_{h-1}\right)=\kappa^{-1} p N(1+\xi p N)^{L-h} \quad \text { for } h \geq 2, \\
\kappa_{\mathrm{cr}}=p N(1+\xi p N)^{L-2} .
\end{gathered}
$$

The stability analysis is performed in the same manner, leading to

$$
\tau=\frac{p N(1+\xi p N)^{L-2}}{\kappa \Gamma^{\prime}\left(\theta_{2}-\theta_{1}\right)} .
$$

Hence, both the entrainment threshold $\kappa_{\mathrm{cr}}$ and the relaxation time $\tau$ decrease dramatically as the backward connectivity $\xi$ gets smaller. The role played by $\xi$ is more significant for the 

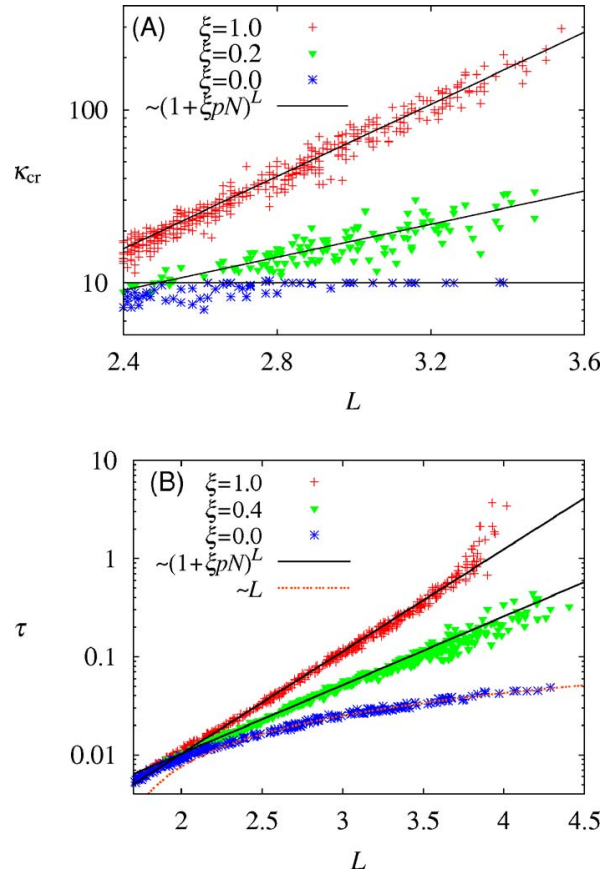

FIG. 8. (Color online) Dependences of (A) the entrainment threshold and (B) the relaxation time on the depth for an ensemble of directionally biased networks. The solid lines are the exponential functions proportional to $(1+\xi p N)^{L}$. The dashed line is a linear function $a L+b$ with appropriate fitting parameters $a$ and $b$. Other parameters are $\kappa=600, p N=10$, and (A) $N=100$ and (B) $N=400$.

networks with a higher hierarchical organization (i.e., with a larger depth $L$ ).

The employed linear approximation is applicable only for large $\xi p N$. For $\xi=0$, the model is, however, exactly solvable without the linear approximation. Since there are no backward connections in this case, perfect phase synchronization inside each shell is possible. Consequently, we get Eqs. (36) and (37) also for $\xi=0$. Trivially, the phase difference $\theta_{h-1}-\theta_{h} \equiv \Delta$ between the neighboring shells is constant for $h>2$, given by the relation $\Gamma(-\Delta)=p N / \kappa$. The stability analysis of this solution is straightforward. The eigenvalue is $\kappa \Gamma^{\prime}(-\Delta) / p N$ (multiplicity $N-N_{1}$ ). For $\kappa>\kappa_{\text {cr }}$, there is at least one pair of solutions, one of which satisfies $\Gamma^{\prime}(-\Delta)$ $<0$. Therefore, for any $\Gamma$, there is always a stable solution for $\kappa>\kappa_{\mathrm{cr}}$. Note that this state has a constant phase gradient and can thus be described as a traveling wave.

Numerical results for directionally biased random networks are displayed in Fig. 8. For the entrainment thresholds [Fig. 8(A)], the theoretical dependence $(1+\xi p N)^{L}$ fits well the numerical data. For the relaxation time [Fig. 8(B)], the theoretical dependence $(1+\xi p N)^{L}$ also fits well the numerical data, except for $\xi=0$ where a linear function fits best. This linear dependence is natural because the solution is wave like and it is thus expected that the time necessarily to transmit information is proportional to the system length (i.e., to the depth $L$ ). Note that the entrainment breakdown occurs near $L=4$ for $\xi=1$.

Similar dependences can be obtained for the weighted networks. To construct them, we first generate an ER network and introduce connections from the pacemaker. We then put
$A_{i j}=\xi$ for all existing backward connections, while keeping the weights of other connections equal to either 0 or 1 . For such networks, we also obtain Eqs. (36)-(38) because the same approximations are applicable.

\section{EVOLUTIONARY OPTIMIZATION}

So far, the relationship between the entrainment threshold and the network depth has been established only for the special kinds of random networks. Therefore, it is natural to ask whether it also holds for arbitrary complex networks. Obviously, we cannot answer it by investigating all possible types of complex networks because their number is too large. Instead, an alternative way is chosen: we shall construct networks with lower entrainment thresholds (and thus better entrainment ability) through an optimization process starting from an arbitrary network architecture and analyze changes of their topological properties in the course of evolution. If these networks actually evolve towards being shallower and closer to a feedforward type, one can conclude that both properties are generally essential for good entrainment ability.

During the evolution, several topological properties shall be monitored. One of them is the depth $L$, which has already been defined. The other property is the backward connectivity $\xi$ defined as the ratio of the total number of backward connections at each trial and that of the initial network. For comparison, we also introduce the forward connectivity $\chi$, given by the ratio between the total number of forward connections in each trial and that of the initial network. The total number of outgoing connections from the first shell, denoted by $n_{\text {out }}$, shall also be monitored.

Two types of optimization algorithms will be employed. The first one is the straightforward optimization where the network structure evolves in such a way that the mean frequency of the whole network becomes closer to that of the pacemaker. In the second algorithm, each oscillator selects its incoming connections in such a way that its own frequency becomes higher. It will be shown that, for both kinds, the evolving network improves its ability to become entrained and, at the same time, indeed becomes less hierarchical and closer to feedforward networks. Note that, concerning biological clocks, the first and second algorithms could imitate, respectively, the evolution process of the neural network of the SCN for a species and the growing process of the neural network for an individual. A more biological algorithm (or learning process) has been employed elsewhere and resulted in similar results [25].

The initial setup is common for both types. The process starts from a random ER network. We put $\phi_{i}(t)=t$ for $i \leq N_{1}$. Throughout the evolution process, the connection pattern from the pacemaker is maintained, so that the first shell is always composed of the oscillators $i \leq N_{1}$. The coupling strength $\kappa$ is fixed far below the entrainment threshold for the initial network. Thus, all the oscillators except in the first shell initially have frequencies close to their natural ones.

The first evolution algorithm. A numerical simulation is run starting from random initial phases. The long-time frequency $\langle\omega\rangle=(T N)^{-1} \sum_{i}\left[\phi_{i}(2 T)-\phi_{i}(T)\right]$, averaged over the 


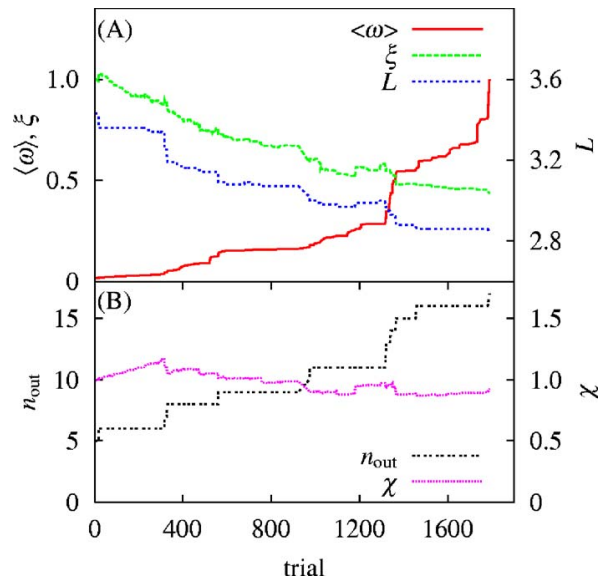

FIG. 9. (Color online) Evolution process under the first algorithm. Parameter values are $N=100, p=0.1, N_{1}=1$, and $\kappa=20$. The depth of the initial random network is $L=3.43$. (A) The solid line is the long-time frequency $\langle\omega\rangle$ averaged over the whole network. The dashed and dotted lines are, respectively, the backward connectivity $\xi$ and the depth $L$. (B) Two other topological quantities are plotted. The solid and dotted lines are, respectively, the total number $n_{\text {out }}$ of outgoing connections from the first shell and the forward connectivity $\chi$.

whole population and for sufficiently long time $T$, is determined. Then, the adjacency matrix $\mathbf{A}$ is mutated. This is done by "rewiring": We choose randomly an existing directed link $\left(A_{i j}=1\right)$ and eliminate it $\left(A_{i j} \rightarrow 0\right)$ and, then, choose randomly a missing link $\left(A_{i^{\prime} j^{\prime}}=0\right.$ where $\left.i^{\prime} \neq j^{\prime}\right)$ and add a new connection there $\left(A_{i^{\prime} j^{\prime}} \rightarrow 1\right)$. After the mutation, a numerical simulation is again run starting from new random initial phases and the new frequency $\langle\omega\rangle^{\prime}$ is determined. If $\langle\omega\rangle^{\prime}$ is closer to 1 than $\langle\omega\rangle$, the mutation is accepted. Otherwise, the network is resetto its structure before the mutation. The iteration process is repeated until the long-time frequency $\langle\omega\rangle$ becomes equal to unity.

A typical dependence of the average frequency and two different topological properties during an evolution is shown in Fig. 9(A). The tendency to decrease both the depth $L$ and the backward connectivity $\xi$ is evident. In most evolutions where the average frequency $\langle\omega\rangle$ has increased, a decrease in $L$ and/or $\xi$ was observed. In Fig. 9(B), the evolution of two other topological properties is shown. We see that the correlation between the feedforward connectivity $\chi$ and the mean frequency $\langle\omega\rangle$ is weak. An increase in $n_{\text {out }}$ indicates the emergence of a hub (i.e., of a node with a large number of outgoing connections), which strongly contributes to decreasing the depth $L$. It is worth noticing that an increase in $\langle\omega\rangle$ does not necessarily imply an increase in $n_{\text {out }}$. Apparently, the entrainment ability relies on finer properties of the network architecture, best characterized by $L$ and $\xi$. In our numerical simulations, we have tried several sets of parameter values and several different initial random networks, always obtaining qualitatively the same results.

The second evolution algorithm. For each iteration step, an oscillator $i$ is randomly chosen. A numerical simulation is run and the long-time frequency of this oscillator, $\omega_{i}=\left[\phi_{i}(2 T)-\phi_{i}(T)\right] / T$ with sufficiently large $T$, is deter-

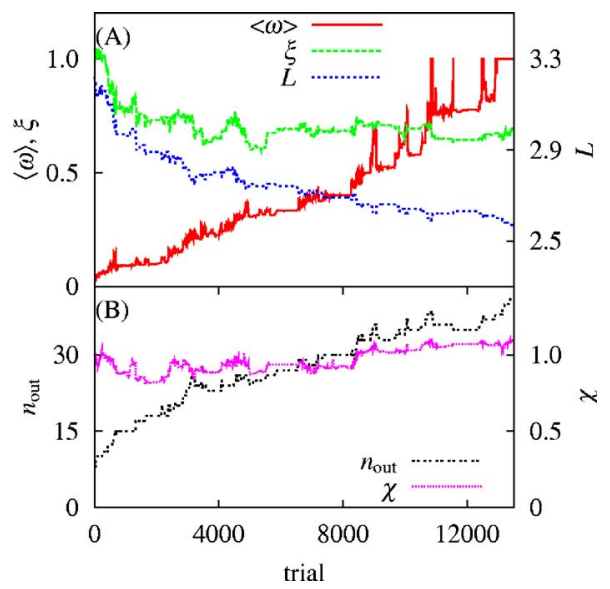

FIG. 10. (Color online) Evolution process under the second algorithm. The depth of the initial random network is $L=3.22$, corresponding to $\kappa_{\mathrm{cr}} \simeq 100$. Parameter values and the definitions of the exhibited lines are the same as in Fig. 9.

mined. Then, a structural mutation of the network is introduced. We choose randomly one existing incoming link of this oscillator $\left(A_{i j}=1\right)$, delete it $\left(A_{i j} \rightarrow 0\right)$, then choose randomly a missing link to this oscillator $\left(A_{i j^{\prime}}=0\right.$ where $\left.j^{\prime} \neq i\right)$, and add a new link there $\left(A_{i j^{\prime}} \rightarrow 1\right)$. After the mutation, a numerical simulation is repeated and the new frequency $\omega_{i}^{\prime}$ of the oscillator $i$ is measured. The mutation is accepted if $\omega_{i}^{\prime}>\omega_{i}$ and rejected otherwise. At the next step, we again randomly choose an oscillator and repeat the same procedure. Thus, in this evolutionary process, the mutation is done according to the individual activities of the oscillators. Note also that, in this evolution algorithm, the total number of incoming connections for each oscillator is maintained constant.

Figure 10(A) displays the typical dependence of the average frequency and two topological properties of the networks during a single evolution. Although the average frequency $\langle\omega\rangle$ of the whole population does not always increase in each iteration step, the network architecture changes during the evolution similar to what has been found for the first algorithm, towards the networks of smaller depth $L$ and smaller backward connectivity $\xi$. In Fig. 10(B), the emergence of a hub and weak correlation between $\langle\omega\rangle$ and $\chi$ are seen, similarly to the results in the first algorithm. It should be emphasized that, in the second algorithm, a globally coordinated network architecture emerges solely through the local optimization rule. The reason is that the oscillators are mutually frequency synchronized in most cases and therefore their individual frequencies usually coincide with the average frequency of the whole population. Hence, the second algorithm also works similarly to the first one.

In both types of algorithms, our numerical results show that the development of the entrainment ability is followed by decreases in the depth $L$ and the backward connectivity $\xi$, suggesting that these two topological properties are the primary factors in determining the entrainment ability. It can be also noticed that the evolving networks tend to develop strong heterogeneity in their outgoing degrees. The depth of an evolving network becomes smaller via an increase in the 
outgoing degrees of nodes in the shallow shells. In this manner, hubs of outgoing connections are formed. On the other hand, the development of small backward connectivity is mainly due to a decrease of the outgoing degrees in the deep shells. Consequently, the heterogeneity of outgoing degrees tends to be larger as the optimization process goes on. ${ }^{2}$ Many real-world networks, including scale-free networks, are known to have strong heterogeneity in their degrees [1]. Our results suggest that evolving networks with good entrainment ability also become strongly heterogeneous.

\section{DISCUSSION OF BIOLOGICAL ASPECTS}

As already mentioned in Sec. I, our study is motivated by the SCN, the tissue orchestrating the circadian rhythms of the whole body in mammals. The SCN is a network of selfsustained oscillators, and each neuron is expected to be described by a limit-cycle oscillator. No matter how these neurons are coupled (including pulse coupled), such a network is generally approximated by phase oscillators coupled through a function of the relative phase difference between each connected pair if coupling is sufficiently weak $[6,22,26-28]$. We thus expect that it is appropriate to model the SCN by coupled phase oscillators. In this section, we discuss the neural network structure of the SCN and its possible roles from the viewpoint of the results of the present investigations.

The SCN is anatomically organized into two groups, often called the "core" subdivision and the "shell" subdivision (see, e.g., Ref. [10]). Only the core subdivision receives photic input and, thus, this part corresponds to the first shell in our model. The shell subdivision corresponds to the rest of the shells in our model. Within and between the core and shell subdivisions, there are several types of intercellular communications which influence circadian oscillations [10]. The pathways (i.e., the network structure) of each type of communication may be different.

From the viewpoint of our results, if the $\mathrm{SCN}$ is constructed so as to reach the best ability for entrainment, the network architecture should be feedforward. This means that the unidirectional connectivity from the core subdivision to the shell subdivision should be found. This is indeed the case for communication via VIP (vasoactive intestinal polypeptide), which is a neurotransmitter released only by neurons in the core subdivision. VIP is one of the leading candidate factors for the synchronization pathway inside the SCN [29]. However, VIP is not the only one communication agent. Communication via GABA ( $\gamma$-aminobutyric acid) is further possible for almost all neurons in the SCN and seems to play a crucial role in achieving synchronization between the core and the shell subdivisions [30]. The GABA connection pattern is not feedforward but bidirectional between these subdivisions. However, remarkably, it has been conjectured from experimental studies that coupling from the shell to the core is weaker than in the other direction [30], suggesting that the network would effectively be close to feedforward one also with respect to the GABA communication.

\footnotetext{
${ }^{2}$ Although there is only little similarity in the models, the evolutionary process described in Ref. [33] has shown a similar result.
}

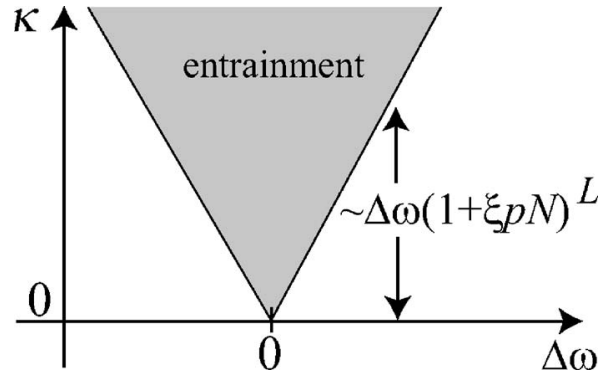

FIG. 11. Schematic representation of the entrainment window, inside which the entrainment occurs. The slopes of the edges of the entrainment window for $\omega>0$ and $\omega<0$ are proportional to $\max \Gamma$ and $\min \Gamma$, respectively.

It is known, moreover, that, although the response of the core structure to a sudden phase shift in the environment is very fast, the response of the shell subdivision to such phase shifts is significantly slower [30,31]. From the viewpoint of our results, it can be conjectured that the slow response of the shell subdivision is due to the more hierarchical, nonfeedforward organization of neurons inside this subdivision. As we have seen, if a network is hierarchical, the response can become very slow even if the first shell is strongly connected to the environment.

It is interesting to ask why the shell subdivision, coupled to the core, actually exists in the SCN and why this subdivision could be more hierarchically organized. One reason may be that such an organization is needed to keep the autonomy of the biological clock. The SCN must be capable to synchronize to the environmental rhythm, but on the other hand, should not be too sensitive to the environmental information. In other words, the SCN must retain both autonomy and adaptivity in a good balance. The hierarchical organization and directionality of the network architecture in the SCN could be useful to reconcile these contradicting requirements.

\section{CONCLUSIONS}

Our main result for the random ER networks and the random directionally biased networks is schematically illustrated in Fig. 11, where $\kappa$ is the nonrescaled coupling strength-i.e., $\kappa$ in the original model (1). The entrainment occurs in a gray region. For the ER networks, this region becomes exponentially smaller for the networks of higher hierarchical organizations (i.e., with larger depth $L$ ). Thus, in practice, the entrainment is possible only for shallow networks. For the directionally biased networks, the entrainment region becomes significantly enlarged for the smaller backward connectivity $\xi$ even if the networks are hierarchical. The feedforward network is the best one for the entrainment. The relaxation time to the entrainment has approximately the same dependence on the network architecture as that of the entrainment threshold. These results are general and hold for a large class of coupled phase-oscillator systems (and thus also of weakly coupled limit-cycle oscillator systems) with attracting couplings. The networks with such topological properties are shown to emerge naturally through different 
kinds of evolutions aimed at increasing the entrainment ability.

\section{ACKNOWLEDGMENTS}

The authors acknowledge fruitful discussions with $\mathrm{K}$. Honma, S. Honma, Y. Kuramoto, N. Masuda, F. Mori., H. Nakao, H. Okamura, and D. Zanette. H.K. acknowledges financial support from the Humboldt Foundation (Germany) and from the Center-of-Excellence Program "Nonlinear Structure via Singularity" in the Department of Mathematics of the Hokkaido University (Sapporo, Japan).

\section{APPENDIX A: ANALYTIC INVESTIGATIONS WITHOUT THE GLOBAL TREE APPROXIMATION}

In Sec. IV, to solve the entrainment problems we have used the heuristic global tree approximation for the random ER networks. By a different method, entrainment problems can be solved directly for the ER network of large size $N \gg 1$ and large degree $p N \gg 1$. Although this systematic derivation is more accurate, it is also more technical and, therefore, we have preferred to present first, in the main part of the article, the analysis based on the global tree approximation. The results of this derivation agree with what we have found before in the global tree approximation and can be viewed as providing further support for it.

We begin by estimating the number $m_{h k}$ of incoming connections leading from all nodes in the $k$ th shell to a node in the $h$ th shell. The network pattern of forward connections is first considered; i.e., $m_{h, h-1}$ for $h \geq 1$ is estimated. By definition, there are $N_{1}$ nodes in the first shell, each of which receives only one forward connection (coming from the pacemaker). We thus have $m_{1,0}=1$. The expected total number of outgoing connections from the nodes in the first shell is $N_{1} p N$ because each node gives typically $p N$ outgoing connections. If $p N N_{1} \ll N$, every outgoing connection almost surely connects to each individually different node outside the first shell. Therefore, in a good approximation, the number $N_{2}$ of nodes in the second shell is $N_{1} p N$ and each node in the second shell receives only one forward connection-i.e., $m_{2,1}=1$. The same property holds up to a certain shell $h^{*}$, where for the first time the total number of outgoing connections from the shell becomes of the order $O(N)$ or largeri.e., $p N N_{h^{*}-1}=o(N)$ and $p N N_{h^{*}} \geq O(N)$. We thus have $N_{h}=N_{1}(p N)^{h-1}(\ll N)$ and $m_{h, h-1}=1$ for $h \leq h^{*}$. In other words, the network pattern of forward connections takes a tree structure in a good approximation up to the shell $h^{*}$. The population $N_{h^{*}+1}$ of the next shell is of the order of $O(N)$. Since $\Sigma_{h \leq h^{*} N_{h}}$ is of $o(N)$, almost all outgoing connections from the shell $h^{*}$ connect to nodes in the shell $h^{*}+1$. Therefore, the expected number of incoming connections from the shell $h^{*}$ to a node in the shell $h^{*}+1$ is $p N N_{h^{*}} / N_{h^{*}+1}$ $\equiv \bar{m}_{h^{*}+1, h^{*}}$. The statistical deviation from this expected number may not be neglected because $\bar{m}_{h^{*}+1, h^{*}}$ is not a large number in general. Since $N_{h^{*}+1}=O(N)$, every node in the network almost surely receives connections coming from the shell $h^{*}+1$, which implies that the rest of the nodes belong to the shell $h^{*}+2$. The expected number $\bar{m}_{h^{*}+2, h^{*}+1}$ of forward connections to a node in the last shell is $p N_{h^{*}+1}$. This is a large number of $O(p N)$, and thus the statistical derivation from the number can be neglected-i.e., $m_{h^{*}+2, h^{*}+1}=p N_{h^{*}+1}$.

Since $L=\sum_{h} h N_{h} / N \simeq\left\{\left(h^{*}+1\right) N_{h^{*}+1}+\left(h^{*}+2\right) N_{h^{*}+2}\right\} / N \simeq h^{*}$ $+1+N_{h^{*}+2} / N$, we get

$$
h^{*}=[L]-1,
$$

where $[L]$ is the integer part of $L$. According to the result in [32], the depth of the ER random network with large size $N$ is estimated as

$$
L \simeq \frac{\ln \left(N / N_{1}\right)-\gamma}{\ln (p N)}+1.5,
$$

where $\gamma \simeq 0.5772$ is the Euler constant.

Next, the structure of backward and intrashell connections is considered. A node in the shell $h$ typically receives $p N_{k}$ connections from nodes in the shell $k(k \geq h)$. The numbers of such connections from the last two shells are of $O(p N)$. Relative statistical deviations from these numbers are of or$\operatorname{der}(p N)^{-1 / 2}$ and thus negligible. We thus obtain $m_{h, k}=p N_{k}$ for $k=h^{*}+1, h^{*}+2$, and $h \leq k$. The number of backward connections from other shells is of $o(p N)$ and thus negligible; i.e., we approximate $m_{h, k}=0$ for $h<k \leq h^{*}$.

Our estimation for the network structure is summarized as follows. The shell populations $N_{h}$ are $N_{1}(p N)^{h-1}$ for $1 \leq h \leq h^{*}$ and of order $O(N)$ for $h=h^{*}+1$ and $h^{*}+2$. The number $m_{h, k}$ of incoming connections of a node in the shell $h$ from the nodes in the shell $k$ is given by $m_{h, h-1}=1$ for $1 \leq h \leq h^{*}$ and by $\bar{m}_{h^{*}+1, h^{*}}=p N N_{h^{*}} / N_{h^{*}+1}, m_{h^{*}+2, h^{*}+1}=p N_{h^{*}+1}$, $m_{h k}=0$ for $k<h-1$ (by definition), $m_{h, k}=p N_{k}$ for $k=h^{*}+1, h^{*}+2$ and $h \leq k$, and $m_{h, k}=0$ for $h<k \leq h^{*}$.

Now we solve the entrainment problem for the network under consideration. We assume that the phase difference $\phi_{i}-\phi_{j}$ between any pair of oscillators in the shells $h \geq 2$ is so small that we may linearize as $\Gamma\left(\phi_{i}-\phi_{j}\right)=\Gamma^{\prime}(0)\left(\phi_{i}-\phi_{j}\right)$. This assumption is justified later [it will be shown that $\left.\left|\phi_{i}-\phi_{j}\right|<O(1 / p N)\right]$.

The dynamics in the shells $h>1$ is considered. We denote the average phase of the oscillators inside the last two shells $h^{*}+1$ and $h^{*}+2$ by $\theta_{\text {edge }}$. Because the number of connections from the last two shells is large, we may approximate the coupling from those shells as

$$
\frac{1}{p N} \sum_{j} A_{i j} \Gamma^{\prime}(0)\left(\phi_{i}-\phi_{j}\right) \simeq \Gamma^{\prime}(0)\left(\phi_{i}-\theta_{\text {edge }}\right),
$$

where $j$ denotes the oscillators inside the last two shells. Since every oscillator in the shell $h \leq h^{*}$ has effectively the same number of incoming connections from each individual shell, a state with phase synchronization inside each shell $h \leq h^{*}$ exists. We denote the phase of the oscillators in the shell $h$ by $\theta_{h}$. Under entrainment (i.e., $\dot{\phi}_{i}=1$ ), the phases of such a state can be found as a solution of algebraic equations

$$
\frac{\kappa}{p N} \Gamma^{\prime}(0)\left(\theta_{h}-\theta_{h-1}\right)+\kappa \Gamma^{\prime}(0)\left(\theta_{h}-\theta_{\text {edge }}\right)=1,
$$

where $2 \leq h \leq h^{*}$. The phase $\phi_{i}^{\text {edge }}$ of the oscillator $i$ inside the last two shells $h^{*}+1$ and $h^{*}+2$ is found by 


$$
\frac{\kappa}{p N} \sum_{j} A_{i j} \Gamma^{\prime}(0)\left(\phi_{i}^{\text {edge }}-\phi_{j}^{h^{*}}\right)+\kappa \Gamma^{\prime}(0)\left(\phi_{i}^{\text {edge }}-\theta_{\text {edge }}\right)=1 .
$$

Averaging Eq. (A5) for all the oscillators inside the last two shells, we get

$$
\frac{\kappa N_{h^{*}}}{N} \Gamma^{\prime}(0)\left(\theta_{\text {edge }}-\theta_{\mathrm{h}^{*}}\right)=1
$$

Note that because of the strong internal coupling inside the last two shells, the oscillators inside the last two shells are almost phase synchronized-i.e., $\phi_{i}^{h} \simeq \theta_{\text {edge }}$ for $h=h^{*}+1$ and $h^{*}+2$. From Eqs. (A4) and (A5), for $2 \leq h \leq h^{*}$ we obtain

$$
\begin{gathered}
\theta_{\text {edge }}-\theta_{h^{*}}=\frac{N}{\Gamma^{\prime}(0) \kappa N_{h^{*}}}, \\
\theta_{h}-\theta_{h-1}=\frac{p N}{\Gamma^{\prime}(0) \kappa}(1+p N)^{h^{*}-h}\left(1+\frac{N}{N_{h^{*}}}\right), \\
\Gamma\left(\theta_{2}-\theta_{1}\right)=\frac{p N}{\kappa}(1+p N)^{h^{*}-2}\left(1+\frac{N}{N_{h^{*}}}\right) .
\end{gathered}
$$

The existence conditions of the entrained solution are $\widetilde{\Gamma}\left(\theta_{1}-\theta_{0}\right) \leq 1$ and $\Gamma\left(\theta_{2}-\theta_{1}\right) \leq 1$. The former is satisfied for sufficiently large $\mu$ (which is our assumption). Thus, for a given network, the existence condition for the entrained solution is $\kappa \leq \kappa_{\mathrm{cr}}$, where

$$
\kappa_{\mathrm{cr}}=\alpha^{-1} p N(1+p N)^{h^{*}-2}\left(1+\frac{N}{N_{h^{*}}}\right) .
$$

Substituting $\kappa_{\text {cr }}$ into $\kappa$ in Eqs. (A7), one can confirm that the phase difference $\theta_{2}-\theta_{\text {edge }}$ is actually at most of $O(1 / p N)$, so that the linear approximation is justified for large $p N$.

In Eq. (A8), because $h^{*}$ increases with the depth $L$, the entrainment threshold approximately increases exponentially with the depth $L$, although the effect of the last term $1+N / N_{h^{*}}$ is unclear. We thus employ further approximations and express the entrainment threshold (A8) as a function of the depth $L$ and the mean degree $p N$. From Eq. (A2), the network size can be expressed as $N=e^{\gamma} N_{1}(p N)^{L-1.5}$. Together with the relations $N_{h^{*}}=N_{1}(p N)^{h^{*}-1}$ and $N \gg N_{h^{*}}$, the last term of Eq. (A8) is approximated as

$$
1+N / N_{h^{*}} \simeq N / N_{h^{*}} \simeq e^{\gamma}(p N)^{L-h^{*}-0.5} .
$$

Because $0.5 \leq L-h^{*}-0.5<1$ from Eq. (A1), we may further approximate it as

$$
e^{\gamma}(p N)^{L-h^{*}-0.5} \simeq e^{\gamma}(1+p N)^{L-h^{*}-0.5}+C,
$$

where $C$ is a small number of order $(p N)^{L-h^{*}-1.5}$. Substituting Eq. (A10) into Eq. (A8) and neglecting the second term $C$ which is much smaller than the first term, we get

$$
\kappa_{\mathrm{cr}} \simeq e^{\gamma} p N(1+p N)^{L-2.5} .
$$

This entrainment threshold agrees well with numerical data without any fitting parameter. Dividing Eq. (A11) by Eq.
(16), we obtain $e^{\gamma}(1+p N)^{-0.5} \equiv c$, which is about $0.67,0.54$, and 0.39 , respectively, for $p N=6,10$, and 20 . These agree well to $c=0.67,0.60$, and 0.45 obtained by numerical fitting (see Fig. 3).

\section{APPENDIX B: ENTRAINMENT THRESHOLD FOR BIDIRECTIONAL NETWORKS}

So far, we have considered only directed networks. However, similar exponential dependences are found also for bidirectional networks. In this appendix, we provide estimation of the entrainment threshold for bidirectional ER networks. Such networks are generated as follows. Independently of any $i$ and $j<i$, we set $A_{i j}=1$ with probability $p$ and $A_{i j}=0$ otherwise and put $A_{j i}=A_{i j}$. Self-connections are forbidden, so that $A_{i i}=0$.

The entrainment threshold is obtained in a similar way as in Sec. IV. We apply a global tree approximation in forward connections (which implies that the pattern of backward connections also take the same structure). Every oscillator then receives only one forward connection. By assuming $p N \gg 1$, every oscillator (except those in the last shell) receives approximately $p N$ backward. connections. ${ }^{3}$ Therefore, the only difference from the ER directed networks is that backward connections come not from the last shell but from the next shell. Thus, we get the following equations:

$$
\mu \widetilde{\Gamma}\left(\theta_{1}-t\right)+\kappa \Gamma\left(\theta_{1}-\theta_{2}\right)=1,
$$

$$
\frac{\kappa}{p N} \Gamma\left(\theta_{h}-\theta_{h-1}\right)+\kappa \Gamma\left(\theta_{h}-\theta_{h+1}\right)=1 \quad \text { for } 2 \leq h<L,
$$

$$
\frac{\kappa}{p N} \Gamma\left(\theta_{L}-\theta_{L-1}\right)=1
$$

Using the approximations available for large $p N$, we obtain

$$
\Gamma\left(\theta_{h}-\theta_{h-1}\right)=\frac{p N\left\{(p N)^{L-h+1}-1\right\}}{\kappa(p N-1)} \quad \text { for } h \geq 2 .
$$

The entrainment thresholds is thus

$$
\kappa_{\mathrm{cr}}=\frac{p N\left\{(p N)^{L-1}-1\right\}}{p N-1} \simeq(p N)^{L-1} .
$$

A stability analysis can also be performed in a same manner as in Sec. IV C. We then get the same relaxation time as Eq. (26). It is thus found that the dependences of the entrainment threshold and the relaxation time are also exponential in the bidirectional networks, but their functional forms are slightly different from those for the directed networks.

\footnotetext{
${ }^{3}$ This approximated network is the same as the Cayley tree. Using some particular phase models, the entrainment solution for the Cayley tree network has been obtained by Yamada [19] and Radicchi and Meyer-Ortmanns [21].
} 
[1] R. Albert and A. L. Barabási, Rev. Mod. Phys. 74, 47 (2002).

[2] S. N. Dorogovtsev and J. F. F. Mendes, Evolution of Networks: From Biological Nets to the Internet and WWW (Oxford University Press, Oxford, 2003).

[3] S. Strogatz, Nature (London) 410, 268 (2001).

[4] A. T. Winfree, J. Theor. Biol. 16, 15 (1967).

[5] A. T. Winfree, The Geometry of Biological Time (Springer, New York, 1980).

[6] Y. Kuramoto, Chemical Oscillations, Waves, and Turbulence (Springer, New York, 1984).

[7] A. Pikovsky, M. Rosenblum, and J. Kurths, Synchronization (Cambridge University Press, Cambridge, England, 2001).

[8] S. C. Manrubia, A. S. Mikhailov, and D. H. Zanette, Emergence of Dynamical Order: Synhronization Phenomena in Complex Systems (World Scientific Singapore, 2004).

[9] S. M. Reppert and D. R. Weaver, Nature (London) 418, 935 (2002).

[10] S. J. Aton and E. D. Herzog, Neuron 48, 531 (2005).

[11] S. Yamaguchi, H. Isejima, T. Matsuo, R. Okura, K. Yagita, M. Kobayashi, and H. Okamura, Science 302, 1408 (2003).

[12] M. Barahona and L. M. Pecora, Phys. Rev. Lett. 89, 054101 (2002).

[13] H. Hong, M. Y. Choi, and B. J. Kim, Phys. Rev. E 65, 026139 (2002).

[14] M. G. Earl and S. H. Strogatz, Phys. Rev. E 67, 036204 (2003).

[15] T. Nishikawa, A. E. Motter, Y. C. Lai, and F. C. Hoppensteadt, Phys. Rev. Lett. 91, 014101 (2003).

[16] T. Ichinomiya, Phys. Rev. E 70, 026116 (2004).
[17] T. Ichinomiya, Phys. Rev. E 72, 016109 (2005).

[18] J. G. Restrepo, E. Ott, and B. R. Hunt, Phys. Rev. E 71, 036151 (2005)

[19] H. Yamada, Prog. Theor. Phys. 108, 12 (2002).

[20] H. Kori and A. S. Mikhailov, Phys. Rev. Lett. 93, 254101 (2004).

[21] F. Radicchi and H. Meyer-Ortmanns, Phys. Rev. E 73, 036218 (2006).

[22] F. Hoppensteadt and E. Izhikevich, Weakly Connected Neural Networks (Springer-Verlag, New York, 1997).

[23] P. Erdös and A. Rényi, Pabl. Math. 6, 290 (1959).

[24] B. Bollobás, Random Graphs (Academic Press, New York, 1985).

[25] N. Masuda and H. Kori (unpublished).

[26] Y. Kuramoto, Physica D 50, 15 (1991).

[27] D. Hansel, G. Mato, and C. Meunier, Neural Comput. 7, 307 (1995).

[28] H. Kori, Phys. Rev. E 68, 021919 (2003).

[29] S. J. Aton, C. S. Colwell, A. J. Haarmar, J. Waschek, and E. D. Herzog, Nat. Neurosci. 8, 476 (2005).

[30] H. Albus, M. J. Vansteensel, S. Michel, G. D. Block, and J. H. Meijer, Curr. Biol. 15, 886 (2005).

[31] M. Nagano, A. Adachi, K. Nakahama, T. Nakamura, M. Tamada, E. Meyer-Bernstein, A. Sehgal, and Y. Shigeyoshi, J. Neurosci. 23, 6141 (2003).

[32] A. Fronczak, P. Fronczak, and J. A. Holyst, Phys. Rev. E 70, 056110 (2004).

[33] J. Ito and K. Kaneko, Phys. Rev. Lett. 88, 028701 (2002). 